\title{
Warning: This keyboard will deconstruct- The role of the keyboard in skilled typewriting
}

\author{
MatThew J. C. CRUMP ANd Gordon D. Logan \\ Vanderbilt University, Nashville, Tennessee
}

\begin{abstract}
Skilled actions are commonly assumed to be controlled by precise internal schemas or cognitive maps. We challenge these ideas in the context of skilled typing, where prominent theories assume that typing is controlled by a well-learned cognitive map that plans finger movements without feedback. In two experiments, we demonstrate that online physical interaction with the keyboard critically mediates typing skill. Typists performed single-word and paragraph typing tasks on a regular keyboard, a laser-projection keyboard, and two deconstructed keyboards, made by removing successive layers of a regular keyboard. Averaged over the laser and deconstructed keyboards, response times for the first keystroke increased by $37 \%$, the interval between keystrokes increased by $120 \%$, and error rate increased by $177 \%$, relative to those of the regular keyboard. A schema view predicts no influence of external motor feedback, because actions could be planned internally with high precision. We argue that the expert knowledge mediating action control emerges during online interaction with the physical environment.
\end{abstract}

We investigated the role of the feel of the keyboard in supporting skilled typing, examining the importance of tactile, haptic, and proprioceptive feedback from the act of typing. Our experiments were partly motivated by dueling intuitions about the feel of the keyboard device in skilled typing. On one hand, the feel of the keyboard seems relatively unimportant. After learning to type on a standard keyboard, we expect our skill to transfer well to a new keyboard when we upgrade our computer or buy a new laptop. After all, computer and laptop keyboards are very similar, with standard-sized $1.9 \times 1.9 \mathrm{~cm}$ keys and a QWERTY key layout, so we do not expect the feel of the new keyboard to drastically disrupt our ability to type. On the other hand, the feel of the keyboard seems essential in typing, as you yourself can demonstrate by typing this sentence without a keyboard by raising your hands and typing in the air. You may not be able to remember key locations without feeling the keyboard underneath your fingers. These dueling intuitions - that manipulations of the feel of a keyboard could prove inconsequential or could drastically disrupt typing ability-inspired an empirical resolution in which we compared typing on regular keyboards with typing on successively deconstructed keyboards, ending with typing on a flat surface.

Although skilled typing has been studied for decades (Cooper, 1983), the role of the keyboard in supporting expert typing has received limited treatment. One line of research was focused on the spatial layout of letter-tokey assignments. Key layout is known to influence initial learning rate but makes little difference to typing rate once the layout is well practiced (Anderson, Mirka, \& Joines,
2007; Liebowitz \& Margolis, 1990; Norman \& Fisher, 1982). However, much less attention has been paid to the role of tactile, haptic, and proprioceptive feedback in supporting expert typing. We are aware of only two studies in which these issues were directly addressed, and these studies demonstrated that typing becomes more error prone when anesthetics are applied to individual fingers (Gordon \& Soechting, 1995; Rabin \& Gordon, 2004).

Given that typing is a motor activity, feedback from the act of typing seems like a necessary component of skilled typing. However, theories of typing have said little about the role of motor feedback (John, 1996; Rumelhart \& Norman, 1982; Salthouse, 1986; Wu \& Liu, 2008). One of the important features of skilled typing is the rapid nature of keystroke execution. In the sample of 356 university students that we have tested in our typing studies, the average typing rate was 67 words per minute (wpm; $S D=19$ ), or about 5-6 keystrokes per second. At this time scale, motor feedback from the keyboard would be too slow to adjust online keystroke execution (Lashley, 1951; Rabin \& Gordon, 2004). As a result, theories of typing assume that keystroke execution is controlled by hierarchical, feedforward processes or schemas. At the top of the hierarchy, word-level processes feed letter-level processes, which in turn trigger action plans for executing individual keystrokes. Schemas are feedforward in the sense that higher-level cognitive processes construct detailed plans for action that are executed by the motor system (Keele, 1968; Lashley, 1951; Newell, 1991; Schmidt, 1975). The plans are supported by a cognitive map of key locations, which allows rapid finger movements to specific key loca-

M. J. C. Crump, matthew.j.crump@vanderbilt.edu 
tions to be planned and executed without feedback from motor processes.

Recent research from our laboratory has challenged the idea that an explicit cognitive map of key locations is precise enough to support accurate typing. Liu, Crump, and Logan (in press) had skilled typists judge direction and distance from one key to another without looking at the keyboard. We found huge errors in judgment, suggesting that typists have poor explicit knowledge of the spatial layout of the keyboard. However, their implicit knowledge was excellent, supporting choice of the correct key locations five to six times per second. This raises the question of the locus of the implicit knowledge. One possibility is that requisite knowledge is strongly internalized and does not require interaction with the keyboard to be expressed. Another possibility is that requisite knowledge is partly externalized (Clark, 2008) and is accessed through the feel of the keyboard that is provided by tactile, haptic, and proprioceptive feedback during the act of typing. In other words, expert knowledge supporting skilled typing may in some sense materialize through physical interaction with the keyboard.

We conducted two experiments to assess the importance of the feel of the keyboard during skilled typing. The first experiment compared typing on deconstructed keyboards that were created by successively dismantling a regular keyboard until we were left with a flat surface. A strongly feedforward view predicts no effect of deconstruction, since movements are planned with respect to a central cognitive map without feedback from the keyboard. A more interactive view predicts strong effects of deconstruction, which systematically removes feedback from tactile, haptic, and proprioceptive sources. In the second experiment, we examined practice with a flat laser device, which projected an image of a keyboard onto a flat surface, to determine whether the disruption in typing skill could be overcome with practice.

\section{EXPERIMENT 1}

In Experiment 1, we tested performance on a regular keyboard (the keys keyboard), a keyboard with the keys removed to expose a set of rubber buttons underneath the keys (the buttons keyboard), a keyboard with the rubber buttons removed to expose a flat plastic surface embedded with electronic circuitry that detects keypresses (the flat keyboard), and a laser keyboard that projects an image of the keyboard on a flat surface (the laser keyboard). Subjects performed discontinuous and continuous typing tasks with the four keyboards. The discontinuous task involved typing single words. We measured first-keypress response time (RT), the interval between successive keystrokes (interkeystroke interval, or IKSI), and error rate. The continuous task involved typing paragraphs. We measured IKSI and accuracy.

If typing is supported by feedforward action plans, performance should remain invariant across the four keyboard types. Alternatively, if typing is influenced by feedback from interacting with the keyboard, performance should be best for the keys condition, which replicates typical typing experience; worse for the buttons condition, in which the feel of the keys was removed but the resistance or give of the keyboard was maintained; and worst for the flat and laser conditions, in which the feel of the keys and the resistance of the keyboard were both removed.

\section{Method}

Subjects. The subjects were 16 students from Vanderbilt University who were recruited for their self-reported ability to type $40 \mathrm{wpm}$. Their skill was confirmed on a typing test. Mean typing speed was $65 \mathrm{wpm}$ (range $=39-89 \mathrm{wpm}$ ). All of the subjects were compensated with $\$ 12$ for $1 \mathrm{~h}$ of participation. All of the subjects had normal or corrected-to-normal vision and spoke English as a first language.

Apparatus and Stimuli. The experiment was conducted on a PC, with a 15-in. SVGA monitor controlled by METACARD software. Typing responses were registered on one of four keyboards: Three keyboards were created by deconstructing a standard dome-switchtype USB QWERTY keyboard in three steps. For the key device, we used the regular keys on the keyboard. Each key was $1.9 \times 1.9 \mathrm{~cm}$. For the button device, we removed the top layer (keys) to expose a rubber membrane spanning the entire keyboard, containing $1.0 \times$ $1.0 \mathrm{~cm}$ bubble-like domes under each key location. Keypresses were registered when a dome was pressed. For the flat device, we removed the rubber membrane to expose two superimposed flat plastic mats containing electronic circuits for each key, registering a keypress when the two sheets were pressed together to close a circuit. Each point of contact was $0.5 \times 0.5 \mathrm{~cm}$. The fourth keyboard was a laser keyboard, which projected an image of the keys on the desktop and registered finger movements on the projected image (Bluetooth Virtual Keyboard, iTech Dynamic, Hong Kong). Each projected key was $1.9 \times 1.9 \mathrm{~cm}$

The USB sampling rate for each keystroke was approximately $125 \mathrm{~Hz}$, and identical across the key, button, and flat keyboards The manufacturer specifications for the laser keyboard claim a USB response rate of 400 characters per minute, which translates to approximately $6.7 \mathrm{~Hz}$. All of the keyboards were clearly labeled with outlines of the keys drawn on the rubber and plastic mats and the letters written within the outlines, and the spacing of the keys was constant across keyboards.

Words for the discontinuous typing task were gathered from the University of South Florida word norms (Nelson, McEvoy, \& Schreiber, 1998). The list was filtered to include words that were four or six characters in length. There were 891 four-letter words (mean word frequency [Kučera \& Francis, 1967] $=150$ per million, range $=94-203$ per million) and 903 six-letter words (mean word frequency $=149$ per million, range $=94-200$ per million); 200 unique words from the four- and six-letter word sets (total $=400$ ) were randomly chosen for each subject.

The continuous typing task involved four paragraphs on the merits of border collies, ranging from 111 to 117 words in length, from Logan and Zbrodoff (1998).

Design and Procedure. In the discontinuous typing task, we presented the subjects with a single word on each trial. There was a total of eight blocks, each containing 50 trials. Each block contained 25 unique four-letter words and 25 unique six-letter words. Each subject responded on four different keyboards. The keyboards were switched every two blocks, and measures of performance for each keyboard were based on two blocks, or 100 trials. Keyboard order (keys, buttons, flat, or laser) was counterbalanced across subjects in four orders, with a balanced Latin square design. The subjects were seated approximately $57 \mathrm{~cm}$ from the computer monitor. At the beginning of each trial, the subjects received a fixation cross $(500 \mathrm{msec})$, followed by a word in the center of the screen in uppercase. The word remained on the screen until the subjects pressed the space bar after typing the word. The typed responses were echoed at the bottom of the screen in lowercase. All letters appeared in black against a gray background. The letters were $8 \mathrm{~mm}$ in height. The 
RTs

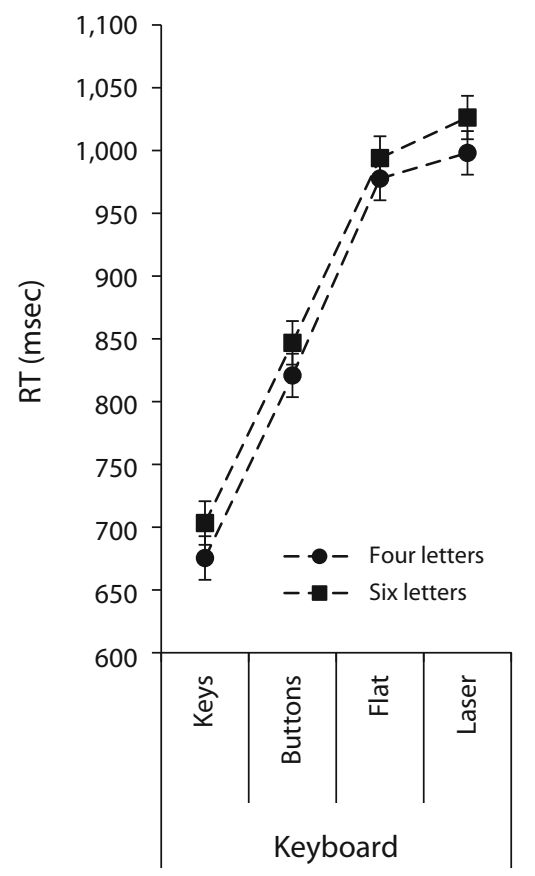

IKSIs

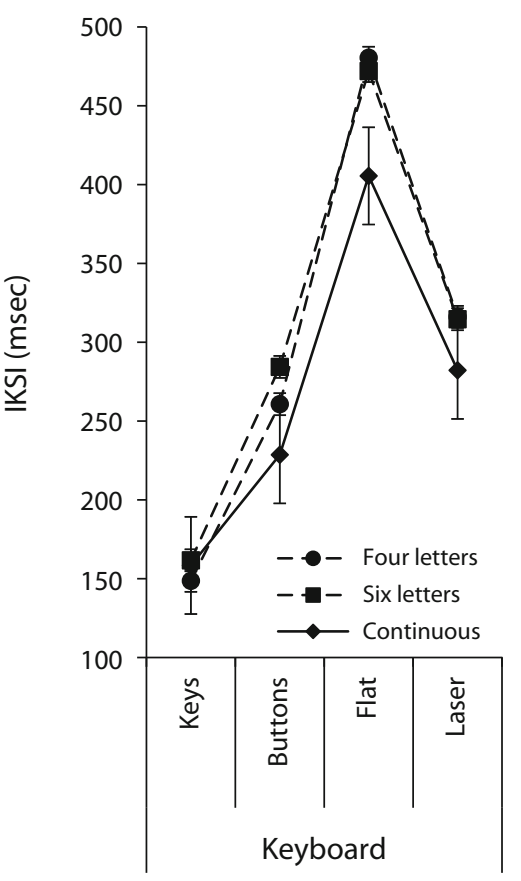

Error Rate

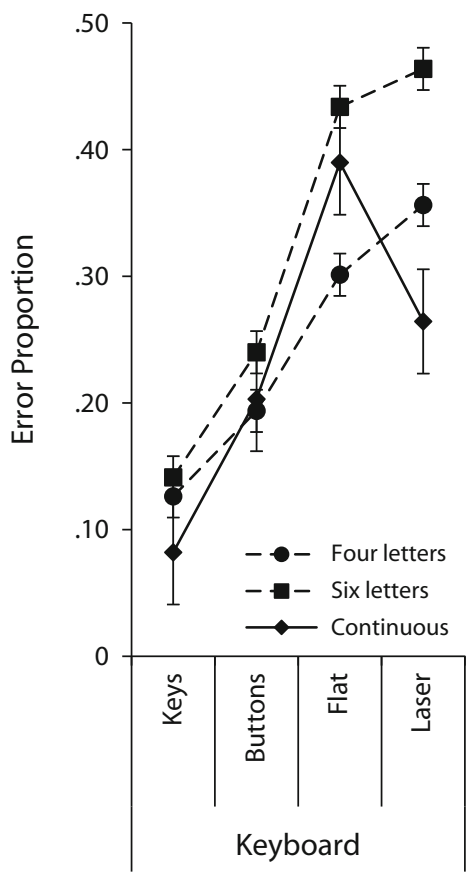

Figure 1. Mean response time (RT) to the first character, interkeystroke interval (IKSI), and error rate in the discontinuous (broken lines) and continuous (solid lines) typing tasks in Experiment 1 as a function of keyboard type (keys, buttons, flat, laser) and word length (four letters vs. six letters). Error bars are Fisher's least significant difference values for $p<.05$, calculated from the interaction between keyboard type and word length in the discontinuous task and from the main effect of keyboard in the continuous task.

subjects were told that the backspace would not function. Thus, all keypress errors were recorded.

The continuous typing task was always presented after the discontinuous typing task. The subjects typed four short paragraphs, one for each keyboard. The paragraphs were randomly assigned to the keyboards for each subject, and keyboard order was counterbalanced with a Latin square design. The paragraph typed with the regular keyboard was used to estimate typing skill in words per minute.

\section{Results and Discussion}

Mean RT, IKSI, and error rates across subjects are presented for each keyboard in Figure 1. Table 1 is a summary of ANOVAs on each measure. For the discontinuous typ- ing task, RT was the time between the presentation of the word and the first keypress. RTs were submitted to an outlier elimination procedure (Van Selst \& Jolicœur, 1994), which removed $3 \%$ of the observations in each cell. Words with errors were excluded from the RT and IKSI analyses. For the continuous typing task, we measured IKSIs and error rates.

All of the measures of performance in the discontinuous typing task were substantially disrupted by deconstructing the keyboard. To evaluate the disruption across keyboards, we calculated Fisher's least significant difference (LSD) for $p<.05$. For RTs, the critical value was $94 \mathrm{msec}$. The

Table 1

Summary Table for ANOVAs in Mean Response Time (RT) to the First Keypress, Interkeystroke Interval (IKSI), and Error Rate in the Discontinuous and Continuous Typing Tasks in Experiment 1

\begin{tabular}{llcccc}
\hline \multicolumn{1}{c}{ Measure } & \multicolumn{1}{c}{ Effect } & $F$ & $d f$ & $M S_{\mathrm{e}}$ & $p$ \\
\hline RT & Keyboard & 20.73 & 3,45 & $34,527.20$ & $<.0001$ \\
& Length & 10.30 & 1,15 & $1,874.86$ & $<.006$ \\
IKSI & Keyboard $\times$ length & $<1$ & & & \\
& Keyboard & 43.34 & 3,45 & $13,023.60$ & $<.0001$ \\
& Length & 1.99 & 1,15 & 700.72 & $<.18$ \\
Error rate & Keyboard $\times$ length & 4.27 & 3,45 & 384.766 & $<.01$ \\
& Keyboard & 15.12 & 3,45 & 0.03522 & $<.0001$ \\
& Length & 66.01 & 1,15 & 0.1815 & $<.0001$ \\
Continuous IKSIs & Keyboard $\times$ length & 10.39 & 3,45 & 0.0022 & $<.0001$ \\
Continuous error rate & Keyboard & 23.24 & 3,45 & $7,497.47$ & $<.0001$ \\
& Keyboard & 19.64 & 3,45 & 0.01337 & $<.0001$ \\
\hline
\end{tabular}


mean RT in the key (standard keyboard) condition was $689 \mathrm{msec}$. The mean RT for the buttons condition was $144 \mathrm{msec}(21 \%)$ longer; that for flat was $296 \mathrm{msec}(43 \%)$ longer; and that for laser was $323 \mathrm{msec}(47 \%)$ longer. All of these differences exceeded the critical value. RTs were significantly longer for the flat and laser conditions than for the buttons condition, but the RTs for the flat and laser conditions were not significantly different. For IKSIs, the critical LSD was $58 \mathrm{msec}$. The mean IKSI in the keys condition was $155 \mathrm{msec}$. The IKSI was $117 \mathrm{msec}(75 \%)$ longer for the buttons condition, $321 \mathrm{msec}(207 \%)$ longer for the flat condition, and $160 \mathrm{msec}(103 \%)$ longer for the laser condition. The IKSI for the flat condition was significantly longer than the IKSI for the buttons and laser conditions, which did not differ significantly from each other. For error rate, the critical LSD value was .09. The mean error rate (probability of at least one error per word) for the keys condition was 0.13 . The mean error rate was $.08(62 \%)$ higher for the buttons condition, .23 (175\%) higher for the flat condition, and .28 (207\%) higher for the laser condition. The mean error rate was significantly higher for the flat and laser conditions than for the buttons condition, but the flat and laser conditions did not differ significantly.

Similar disruptions were seen in the continuous typing task. For IKSI, the critical LSD value was $62 \mathrm{msec}$. The mean IKSI for the keys condition was $158 \mathrm{msec}$, which was very close to the mean IKSI in discontinuous typing. The mean IKSI was $70 \mathrm{msec}(44 \%)$ longer for the buttons condition, $247 \mathrm{msec}(156 \%)$ longer for the flat condition, and $124 \mathrm{msec}(78 \%)$ longer for the laser condition. The buttons and laser conditions did not differ significantly, but the mean IKSI for the flat condition was significantly longer than that for the buttons and laser conditions. For error rate, the critical LSD was .08. The mean error rate (per word) for the keys condition was .08. The mean error rate was $0.12(147 \%)$ higher for the buttons condition, $0.31(325 \%)$ higher for the flat condition, and $0.18(222 \%)$ higher for the laser condition. The buttons and laser conditions were not significantly different, but the mean error rate for the flat condition was significantly higher than that for the buttons and laser conditions.

These large disruptions are not consistent with a strong feedforward view of typing. Performance was best when the subjects interacted with the keyboard on which their typing skill was usually expressed. Performance was progressively worse the more the keyboard differed from a regular one. The flat keyboards produced the worst performance. These findings demonstrate a substantial role for online tactile, haptic, and proprioceptive feedback in supporting the fluidity of typing skill.

\section{EXPERIMENT 2}

Experiment 1 demonstrated that the feel of the keyboard is a crucial component of typing skill. In Experiment 2, we asked whether the disruptions in performance were due to the novelty of typing on unfamiliar keyboards. The subjects in Experiment 1 had only 100 trials with each keyboard. In Experiment 2, we investigated whether performance with the laser-projection keyboard would improve with further practice. Subjects were given 300 extra trials of practice (for a total of 400 trials across four blocks). As in Experiment 1, every word was unique. Crump and Logan (in press) found substantial reductions in IKSI with 480 trials of practice on a laser keyboard, but their subjects typed the same 20 words throughout practice (also see Roeber, Bacus, \& Tomasi, 2003). In Experiment 2, we asked whether general benefits might result from practice, so we presented a unique word on each trial. For comparison, we had the subjects type 50 unique words on a regular keyboard before and after training.

\section{Method}

Subjects. Subjects were 16 students from Vanderbilt University recruited for their self-reported ability to type $40 \mathrm{wpm}$ or more. Mean speed on the typing test was $65 \mathrm{wpm}($ range $=48-94 \mathrm{wpm})$. All of the subjects were compensated $\$ 12$ for $1 \mathrm{~h}$ of participation. All of the subjects had normal or corrected-to-normal vision and spoke English as a first language.

Apparatus and Stimuli. We employed the same computer apparatus, keys (regular), and laser-projection keyboards as in Experiment 1 . Four- and six-letter words were drawn randomly for each subject from the same word lists described in Experiment 1.

Design and Procedure. We employed the same discontinuous typing task reported in Experiment 1, except that the subjects were only given the keys and laser-projection keyboards. There were six blocks of 100 trials (50 four-letter words, 50 six-letter words). In the first and last blocks, the subjects were given the regular keyboard. The subjects were tested on the laser-projection keyboard in Blocks 2-5. After completing the discontinuous typing task, the subjects performed the continuous typing task from Experiment 1, typing two paragraphs, one on the regular keyboard, and one on the laser keyboard.

\section{Results}

Mean RTs, IKSIs, and error rates as a function of word length and block are depicted in Figure 2. The results from repeated measures ANOVAs on each measure are reported in Table 2. Performance was substantially disrupted during the middle four blocks, in which the subjects typed on the laser keyboard. For Blocks 1 and 6, the mean RT was $654 \mathrm{msec}$, the mean IKSI was $150 \mathrm{msec}$, and the mean error rate was .05. For Blocks 2-5, the mean RT increased by $193 \mathrm{msec}(30 \%)\left[F(1,75)=234.20, M S_{\mathrm{e}}=\right.$ $6,805.19, p<.01]$, the mean IKSI increased by $144 \mathrm{msec}$ $(96 \%)\left[F(1,75)=239.28, M S_{\mathrm{e}}=3,674.14, p<.01\right]$, and the mean error rate increased by $.13(262 \%)[F(1,75)=$ $\left.138.98, M S_{\mathrm{e}}=0.00512, p<.01\right]$. There was no evidence of improvement over the middle four blocks, in which the subjects practiced typing on the laser keyboard. Tests of linear trends over Blocks $2-5$ showed no significant change in RT $\left[F(1,75)<1, M S_{\mathrm{e}}=6,805.19\right]$, a small amount of slowing in IKSI $\left[F(1,75)=3.28, M S_{\mathrm{e}}=3,674.14, p<\right.$ $.08]$, and a significant increase in error rate $[F(1,75)=$ $\left.5.88, M S_{\mathrm{e}}=0.00512, p<.05\right]$.

The finding that 400 trials of practice on the laser keyboard were not sufficient to improve performance suggests that the results of Experiment 1 are not merely due to the novelty of the typing instrument (cf. Norman \& Fisher, 1982). Instead, disruptions to performance from deconstructing the keyboard are substantial and persis- 
RTs

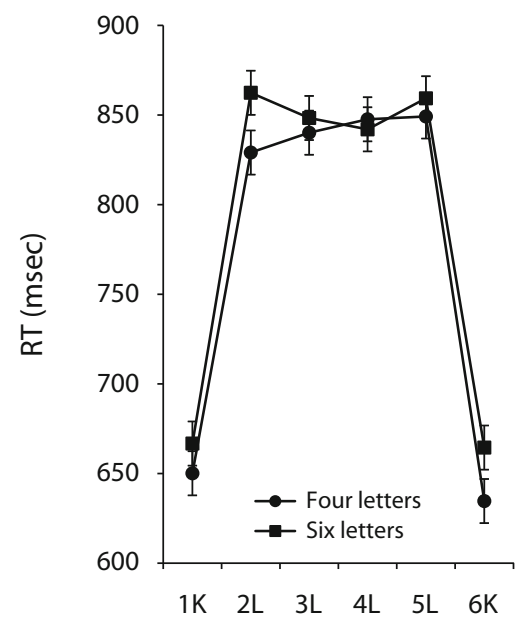

IKSIs

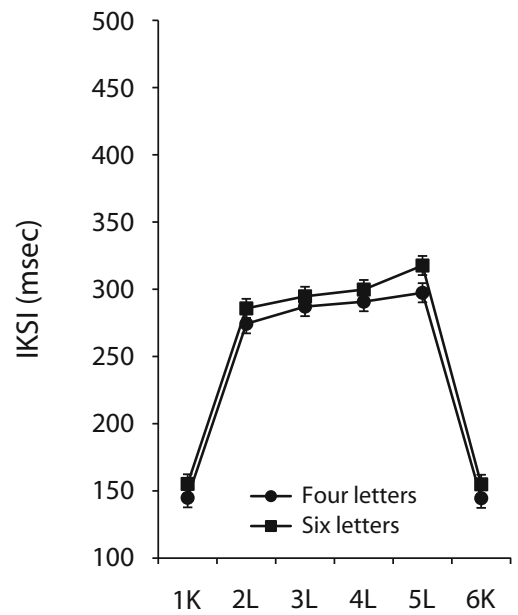

Error Rate

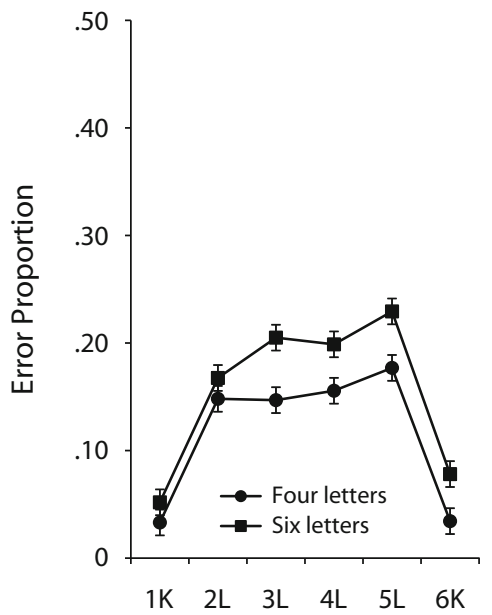

Figure 2. Mean response time (RT) to the first character, interkeystroke interval (IKSI), and error rate for the discontinuous typing task in Experiment 2 as a function of block (1-6) and word length (four letters vs. six letters). In Blocks 1 and 6, we used the regular keyboard; in Blocks 2-5, we used the laser keyboard. Error bars are Fisher's least significant difference values for $p<.05$, calculated from the interaction between block and word length.

Table 2

Summary Table for ANOVAs in Mean Response Time (RT) to the First Keypress, Interkeystroke Interval (IKSI), and Error Rate in the Discontinuous and Continuous Typing Tasks in Experiment 2

\begin{tabular}{llrrrl}
\hline Measure & \multicolumn{1}{c}{ Effect } & \multicolumn{1}{c}{$F$} & $d f$ & $M S_{\mathrm{e}}$ & \multicolumn{1}{c}{$p$} \\
\hline RT & Block & 46.92 & 5,75 & 6805.19 & $<.0001$ \\
& Length & 10.81 & 1,15 & 1046.08 & $<.005$ \\
& Block $\times$ length & 1.37 & 5,75 & 1222.09 & $<.25$ \\
IKSI & Block & 48.51 & 5,75 & 3674.14 & $<.0001$ \\
& Length & 3.90 & 1,15 & 6371.02 & $<.07$ \\
& Block $\times$ length & $<1$ & & & \\
Error rate & Block & 29.19 & 5,45 & 0.00512 & $<.0001$ \\
& Length & 66.29 & 1,15 & 0.0011 & $<.0001$ \\
& Block $\times$ length & 2.44 & 5,45 & 0.00091 & $<.05$ \\
\hline
\end{tabular}

tent. The present results also suggest that the practice effects in Crump and Logan (in press) were specific to the repeated items.

\section{GENERAL DISCUSSION}

The present experiments resolve the dueling intuitions about the role of the keyboard in typing. We may adapt to a new computer relatively easily, but deconstructing the keyboard to remove the feel and resistance of the keys dramatically impaired typing in both experiments. The contrast between the regular keyboard and the buttons keyboard suggests that the feel of the keys is important, and the contrast between the buttons, flat, and laser keyboards suggests that the resistance of the keys is important as well. However, our deconstructed keyboards differed from the standard keyboard in other ways, so it is difficult to isolate single factors responsible for the disruption.

The buttons were smaller than the regular keys, and the sensitive regions of the flat keyboard were smaller than the buttons, and smaller targets take longer to hit (Fitts, 1954). However, the keys on the laser keyboard were the same size as the keys on the regular keyboard, yet the laser keyboard produced RTs like the flat keyboard, IKSIs like the button keyboard, and the highest error rate in discontinuous typing, so size cannot be everything. The angle of the laser keyboard was flatter than that of the regular keyboard, but the angles of the button and flat keyboards were the same as that of the regular keyboard. The subjects could not rest their fingers on the home row of the flat and laser keyboards without triggering inadvertent keystrokes, whereas they could on the regular and button keyboards, and this may have contributed to the disruption. Indeed, the mean IKSIs were about the same on the laser keyboard and the button keyboard, suggesting that resting on the home row was not so important once the fingers were in motion. To test the importance of the feel of the keys in aligning the fingers on the home row, we had 61 typists place their hands on a blank piece of paper as if they were on the home row, and we traced the outline of their fingers. The outline was curved instead of straight (mean discrepancy from straight $=12.5 \mathrm{~mm} ; S E=0.6 \mathrm{~mm}$ ), suggesting that feedback from the keys is important in aligning the fingers with the home row. 
The importance of the keyboard in the expression of typing skill suggests that interacting with the keyboard is as important to typing as planning to interact with the keyboard is (also see Liu et al., in press). This conclusion challenges current theories of typing, which postulate feedforward action plans that control keystroke execution (John, 1996; Rumelhart \& Norman, 1982; Wu \& Liu, 2008). These theories do not deny the importance of feedback, but they do not describe a role for it in the computations that control typing. Extending these theories to include feedback from the keyboard during typing is an important step for future research.

The disruptive effects of deconstructing the keyboard are consistent with recent perspectives on embodied and extended cognition (Barsalou, 1999; Clark, 2008; Glenberg, 1997; Wilson, 2002), which emphasize the importance of motor processes in cognition and the importance of the environment in cognitive control. The role of the standard keyboard in supporting skilled typing suggests that the spatial map of key locations may be the keyboard itself. Without feedback from the keyboard, the cognitive map is not precise enough to support accurate typing (Liu et al., in press). More generally, the results support efforts to integrate research on cognition with research on motor control (Rosenbaum, 2005). Typewriting may be a useful paradigm for integration, because it recruits a wide variety of cognitive and motor processes (Salthouse, 1986).

\section{AUTHOR NOTE}

This research was supported by Grant BCS 0646588 from the National Science Foundation. Correspondence concerning this article should be addressed to M. J. C. Crump, Department of Psychology, Vanderbilt University, Nashville, TN 37203 (e-mail: matthew.j.crump @.vanderbilt.edu).

\section{REFERENCES}

Anderson, A. M., Mirka, G. A., \& Joines, S. M. B. (2007). Learning rate analysis of alternative keyboards. Human Factors \& Ergonomics Society Annual Meeting Proceedings, Industrial Ergonomics, 5, 874-878.

Barsalou, L. W. (1999). Perceptual symbol systems. Behavioral \& Brain Sciences, 22, 577-660.

Clark, A. (2008). Supersizing the mind: Embodiment, action, and cognitive extension. Oxford: Oxford University Press.

Cooper, W. E. (1983). Cognitive aspects of skilled typewriting. New York: Springer.

Crump, M. J. C., \& Logan, G. D. (in press). Episodic contributions to sequential control: Learning from a typist's touch. Journal of Experimental Psychology: Human Perception \& Performance.

FitTs, P. M. (1954). The information capacity of the human motor system in controlling the amplitude of movement. Journal of Experimental Psychology, 47, 381-391.
Glenberg, A. M. (1997). What memory is for. Behavioral \& Brain Sciences, 20, 1-55.

Gordon, A. M., \& Soechting, J. F. (1995). Use of tactile afferent information in sequential finger movements. Experimental Brain Research, 107, 281-292.

JoHn, B. E. (1996). TYPIST: A theory of performance in skilled typing. Human-Computer Interaction, 11, 321-355.

KeELE, S. W. (1968). Movement control in skilled motor performance. Psychological Bulletin, 70, 387-403.

KuČERA, H., \& FRANCIS, W. N. (1967). Computational analysis of presentday American English. Providence, RI: Brown University Press.

LASHLEY, K. S. (1951). The problem of serial order in behavior. In L. A. Jeffress (Ed.), Cerebral mechanisms in behavior (pp. 112-131). New York: Wiley.

Liebowitz, S. J., \& Margolis, S. E. (1990). The fable of the keys. Journal of Law \& Economics, 33, 1-25.

LiU, X., Crump, M. J. C., \& Logan, G. D. (in press). Do you know where your fingers have been? Explicit knowledge of the spatial layout of the keyboard in skilled typists. Memory \& Cognition.

Logan, G. D., \& ZBrodofF, N. J. (1998). Stroop-type interference: Congruity effects in color naming with typewritten responses. Journal of Experimental Psychology: Human Perception \& Performance, 24, 978-992.

Nelson, D. L., McEvoy, C. L., \& Schreiber, T. A. (1998). The University of South Florida word association, rhyme, and word fragment norms. Retrieved February 18, 2008, from www.usf.edu/ FreeAssociation/.

Newell, K. M. (1991). Motor skill acquisition. Annual Review of Psychology, 42, 213-237.

Norman, D. A., \& Fisher, D. (1982). Why alphabetic keyboards are not easy to use: Keyboard layout doesn't matter much. Human Factors, 24, 509-519.

Rabin, E., \& Gordon, A. M. (2004). Tactile feedback contributes to consistency of finger movements during typing. Experimental Brain Research, 155, 362-369. doi:10.1007/s00221-003-1736-6

Roeber, H., Bacus, J., \& Tomasi, C. (2003). Typing in thin air: The Canesta projection keyboard-A new method of interaction with electronic devices. In CHI '03 extended abstracts on human factors in computing systems (pp. 712-733). New York: Association for Computing Machinery.

Rosenbaum, D. A. (2005). The Cinderella of psychology: The neglect of motor control in the science of mental life and behavior. American Psychologist, 60, 308-317. doi:10.1037/0003-066X.60.4.308

Rumelhart, D. E., \& Norman, D. A. (1982). Simulating a skilled typist: A study of skilled cognitive-motor performance. Cognitive Science, 6, 1-36.

Salthouse, T. A. (1986). Perceptual, cognitive, and motoric aspects of transcription typing. Psychological Bulletin, 99, 303-319.

ScHMIDT, R. A. (1975). A schema theory of discrete motor skill learning. Psychological Review, 82, 225-260.

Van Selst, M., \& Jolicceur, P. (1994). A solution to the effect of sample size on outlier elimination. Quarterly Journal of Experimental Psychology, 47A, 631-650.

Wilson, M. (2002). Six views of embodied cognition. Psychonomic Bulletin \& Review, 9, 625-636.

Wu, C., \& LiU, Y. (2008). Queuing network model of transcription typing. ACM Transactions on Computer-Human Interaction, 15, Article 6.

(Manuscript received February 25, 2009 . revision accepted for publication December 2, 2009.) 